\title{
The SportSense User Interface for Holistic Tactical Performance Analysis in Football
}

\author{
Philipp Seidenschwarz ${ }^{1,2}$ \\ philipp.seidenschwarz@bfh.ch \\ Martin Rumo ${ }^{2}$ \\ martin.rumo@bfh.ch \\ ${ }^{1}$ Dept. of Mathematics and Computer Science \\ University of Basel \\ Basel, Switzerland
}

Adalsteinn Jonsson ${ }^{1}$

a.jonsson@stud.unibas.ch

Lukas Probst ${ }^{1}$

lukas.probst@unibas.ch

\author{
Michael Plüss ${ }^{1}$ \\ m.pluess@unibas.ch
}

Heiko Schuldt ${ }^{1}$
heiko.schuldt@unibas.ch

\begin{abstract}
In today's team sports, the effective and user-friendly support of analysts and coaches in analyzing their team's tactics is essential. In this paper, we present an extended version of SPORTSENSE, a tool for searching in sports video by means of sketches, for creating and visualizing statistics of individual players and the entire team, and for visualizing the players' off-ball movement. SportSEnse has been developed in close collaboration with football coaches.
\end{abstract}

\section{CCS CONCEPTS}

- Information systems $\rightarrow$ Information systems applications; Multimedia and multimodal retrieval; $\bullet$ Humancentered computing $\rightarrow$ Graphical user interfaces.

\section{KEYWORDS}

Data-Driven Analysis; Video Analysis; Sketch-based Video Retrieval; Quantitative and Qualitative Match Analysis; SpatioTemporal Data; Graphical User Interfaces.

\section{ACM Reference Format:}

Philipp Seidenschwarz, Adalsteinn Jonsson, Michael Plüss, Martin Rumo, Lukas Probst, and Heiko Schuldt. 2020. The SportSense User Interface for Holistic Tactical Performance Analysis in Football. In 25th International Conference on Intelligent User Interfaces Companion (IUI '20 Companion), March 17-20, 2020, Cagliari, Italy. ACM, New York, NY, USA, 2 pages. https://doi.org/10.1145/3379336. 3381473

Permission to make digital or hard copies of part or all of this work for personal or classroom use is granted without fee provided that copies are not made or distributed for profit or commercial advantage and that copies bear this notice and the full citation on the first page. Copyrights for thirdparty components of this work must be honored. For all other uses, contact the owner/author(s).

IUI '20 Companion, March 17-20, 2020, Cagliari, Italy

(c) 2020 Copyright held by the owner/author(s).

ACM ISBN 978-1-4503-7513-9/20/03.

https://doi.org/10.1145/3379336.3381473
${ }^{2}$ Centre of Tech. in Sports \& Medicine Bern Univ. of Applied Sciences Nidau-Biel, Switzerland

\section{INTRODUCTION}

The importance of tactical performance analysis in team sports and especially in football has enormously increased over the last years. However, this is a very complex and timeintensive task for coaches and analysts as they have to search for specific video scenes, handle large data sets, and identify key performance indicators to be well prepared for the next matches. In this paper, we present the SPORTSEnSE UI, an analysis tool which allows a coach or analyst to search for video scenes by means of hand-drawn sketches of player interactions, as well as calculating statistics on-the-fly [2, 3, 5]. Additionally, off-ball movements of the players can be visualized. This helps a coach or an analyst to holistically analyze tactical elements and to evaluate player and team performances in a flexible and time-efficient manner.

\section{SPORTSENSE}

The SportSense user interface has been targeted to users with rather low IT affinity. The main rationale behind the UI and especially of its drawing area is to mimic the traditional tactic board coaches and analysts are familiar with.

The SportSEnse UI consists of four main components: (i) a video area (upper left part in Fig. 1), where the video source is displayed, (ii) a drawing area (upper middle part in Fig. 1), where the user can define areas and draw sketches to search for events and patterns of events, (iii) a filter area (upper right and middle layer in Fig. 1), where various filters can be set to further define the search, and (iv) a timeline (lower part of Fig. 1), where the results of the search are visualized.

\section{Functionalities}

SPORTSENSE supports three different sketch-based retrieval methods. With Region Queries, a user can define a region on the schematic pitch in the drawing area where specific events occurred. With Event Cascades, the search for patterns of events is supported. A user can define an area with a region query and search for events either happened before the 


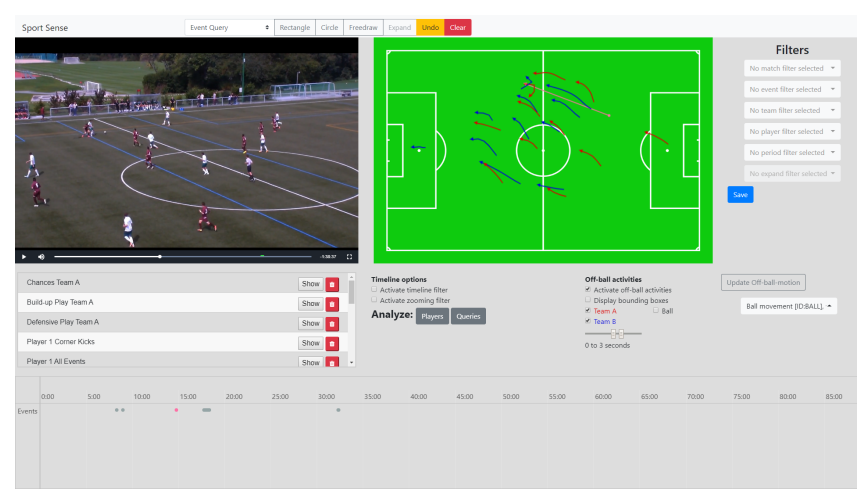

Figure 1: SportSense UI with visualized off-ball movements

results of the region query (Reverse Event Cascade) or afterwards (Forward Event Cascade). The third retrieval method is called Motion Path. A user can sketch the path of a player or the ball on the drawing area. For all retrieval modes, clicking on one of the retrieved results will display the corresponding video scene in the video area of the SPORTSENSE UI.

An important element of tactical performance analysis considers off-ball movements of the players and teams in specific situations. SPORTSENSE supports such analyzes by allowing users to select an event of interest; then, the trajectories of the players and the ball are visualized in the UI. With a slider, the time interval for which the trajectories are retrieved and displayed can be varied.

Statistics and key performance indicators are other core elements in team performance analysis. These can be used, for instance, to justify decisions to players, or to get a confirmation of the subjective impressions of training or match performances. SPORTSENSE allows a user to calculate on-thefly statistics by saving queries and compare them with respect to various parameters (see Fig. 2). Additionally, coaches or analysts can compare historical player data and compare different players with respect to various parameters.

\section{SportSense in Action}

SPORTSENSE relies on position data (ball and players) as well as event data. Additionally, videos from the corresponding matches are needed for the full functionality of the system. Interaction with SPORTSENSE includes drawing sketches, saving the results and analyzing them either qualitatively by watching the video scenes or quantitatively by calculating statistics. Additionally, also player statistics can be compared.

\section{RELATED WORK}

Previous work on sketch-based retrieval has mainly addressed football [4], but also other team sports (e.g., basketball [6]). Other approaches focus on the visualization of complex concepts of football, e.g., pressure [1] or dominant regions [7]. However, to the best of our knowledge, there is no system

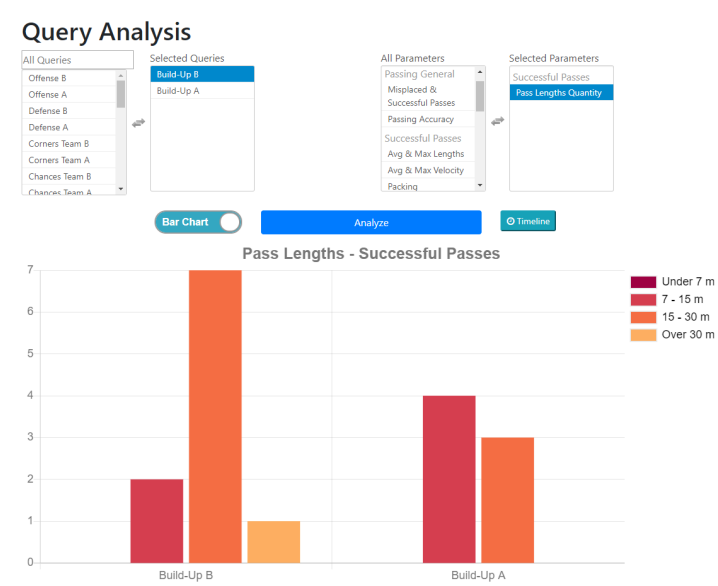

Figure 2: SportSense UI with visualized statistics

that covers such a broad spectrum of tactical performance analyses as SportSense.

\section{CONCLUSION}

SPORTSENSE is a powerful tool that supports coaches and analysts in their time-intensive task of tactical performance analysis. It provides various options to search for events and patterns of events in the corresponding video scenes, it is able to visualize off-ball movements, and it allows users to calculate on-the-fly statistics for the comparison of performances. It is available under an open source license (https://github.com/ sportsense). In our future work we plan to integrate physical performance data, which will open a new set of analyzes.

\section{ACKNOWLEDGMENTS}

This work has been partly supported by the Hasler Foundation (project STREAMTeam, contract no. 16074).

\section{REFERENCES}

[1] Gennady Andrienko et al. 2017. Visual analysis of pressure in football. Data Mining and Knowledge Discovery 31, 6 (May 2017), 1793-1839.

[2] Lukas Probst et al. 2018. Integrated Real-Time Data Stream Analysis and Sketch-Based Video Retrieval in Team Sports. In Proc. of the 2018 IEEE International Conference on Big Data. IEEE, 548-555.

[3] Lukas Probst et al. 2018. SportSense: User Interface for Sketch-Based Spatio-Temporal Team Sports Video Scene Retrieval. In Proc. of the 1st Workshop on User Interface for Spatial and Temporal Data Analysis.

[4] Keven Richly. 2018. Leveraging Spatio-Temporal Soccer Data to Define a Graphical Query Language for Game Recordings. In Proc. of the 2018 IEEE International Conference on Big Data. IEEE, 3456-3463.

[5] Philipp Seidenschwarz et al. 2019. Combining Qualitative and Quantitative Analysis in Football with SportSense. In Proc. of the 2nd International Workshop on Multimedia Content Analysis in Sports. ACM, 34-41.

[6] Long Sha et al. 2018. Interactive sports analytics: An intelligent interface for utilizing trajectories for interactive sports play retrieval and analytics. Transactions on Computer-Human Interaction 25, 2 (2018).

[7] Manuel Stein et al. 2017. Bring It to the Pitch: Combining Video and Movement Data to Enhance Team Sport Analysis. Transactions on Visualization and Computer Graphics 24, 1 (2017), 13-22. 